\title{
Some remarks on spatial uniformity of solutions of reaction-diffusion PDEs
}

\author{
Zahra Aminzare* Eduardo D. Sontag ${ }^{\dagger}$
}

\begin{abstract}
In this paper, we present a condition which guarantees spatial uniformity for the asymptotic behavior of the solutions of a reaction diffusion partial differential equation (PDE) with Neumann boundary conditions in one dimension, using the Jacobian matrix of the reaction term and the first Dirichlet eigenvalue of the Laplacian operator on the given spatial domain. The estimates are based on logarithmic norms in non-Hilbert spaces, which allow, in particular for a class of examples of interest in biology, tighter estimates than other previously proposed methods.
\end{abstract}

Key words: Spatial uniformity, Synchronization, Contraction, Reaction diffusion PDEs, Logarithmic norm, Logarithmic Lipschitz constant.

\section{Introduction}

In this paper we study asymptotic behavior of the solutions of reaction diffusion PDE systems of the general form:

$$
\begin{gathered}
\frac{\partial u_{1}}{\partial t}(\omega, t)=F_{1}(u(\omega, t), t)+d_{1}(t) \Delta u_{1}(\omega, t) \\
\vdots \\
\frac{\partial u_{n}}{\partial t}(\omega, t)=F_{n}(u(\omega, t), t)+d_{n}(t) \Delta u_{n}(\omega, t),
\end{gathered}
$$

subject to the Neumann boundary condition:

$$
\frac{\partial u_{i}}{\partial \mathbf{n}}(\xi, t)=0, \quad \forall \xi \in \partial \Omega, \quad \forall i=1, \ldots, n,
$$

or subject to the Dirichlet boundary condition:

$$
u_{i}(\xi, t)=0, \quad \forall \xi \in \partial \Omega, \quad \forall i=1, \ldots, n,
$$

* The Program in Applied and Computational Mathematics, Princeton University, Princeton, NJ 08544-1000 USA. Email: aminzare@math.princeton.edu.

${ }^{\dagger}$ Department of Mathematics, Rutgers University, Piscataway, NJ 08854-8019 USA.Email: eduardo.sontag@rutgers.edu. 
or, in vector form:

$$
\frac{\partial u}{\partial t}(\omega, t)=F(u(\omega, t), t)+D(t) \Delta u(\omega, t), \quad \omega \in \Omega
$$

where we assume

- $\Omega$ is an open and bounded subset of $\mathbb{R}^{m}$ with smooth boundary $\partial \Omega$ and outward normal $\mathbf{n}$.

- $u(\omega, t)=\left(u_{1}(\omega, t), \ldots, u_{n}(\omega, t)\right)^{T}$, where for any $i, u_{i}: \bar{\Omega} \times[0, \infty) \rightarrow \mathbb{R}$ is twice continuously differentiable in the first argument and continuously differentiable in the second argument.

- $F: V \times[0, \infty) \rightarrow \mathbb{R}^{n}$ is a Lipschitz (in its first argument) vector field with components $F_{i}$ :

$$
F(x, t)=\left(F_{1}(x, t), \ldots, F_{n}(x, t)\right)^{T},
$$

for some functions $F_{i}: V \times[0, \infty) \rightarrow \mathbb{R}$, where $V$ is a convex subset of $\mathbb{R}^{n}$.

- $D(t)=\operatorname{diag}\left(d_{1}(t), \ldots, d_{n}(t)\right)$, where for each $i, d_{i}(t) \geq 0$ is a continuous function of $t$. The matrix $D(t)$ is called the diffusion matrix.

- $\Delta u=\left(\Delta u_{1}, \ldots, \Delta u_{n}\right)^{T}$, where $\Delta=\nabla \cdot \nabla$ is the Laplacian operator defined by $\Delta v=\sum_{i=1}^{m} \frac{\partial^{2} v}{\partial \omega_{i}^{2}}$ for $v=v\left(\omega_{1}, \ldots, \omega_{m}\right)$.

In [1], we identified a class of reaction diffusion equations with the property that any two solutions converge to each other exponentially, i.e.,

$$
\|(u-v)(\cdot, t)\| \leq e^{-c t}\|(u-v)(\cdot, 0)\|
$$

for some norm $\|\cdot\|$ and some positive constant $c$ which only depends on the reaction part $F$; we review the main result of [1] in Theorem 1 below.

In this paper, we are interested in a class of reaction diffusion equations with the property that every solution converges to a spatially uniform solution. We will see (Remark 2 below) that any system in the former class belongs to the latter class. But there are some systems (such as the so-called "Goodwin oscillator" model that is a standard example in molecular biology) that do not belong to the former class but do belong to the latter class. Motivated by these systems, we provide a condition of the reaction part $F$ as well as the diffusion matrix $D$ and the structure of $\Omega$, that guarantees spatial uniformity for the asymptotic behavior of the solutions of a reaction diffusion PDE with Neumann boundary conditions.

The convergence to uniform solutions in reaction diffusion partial differential equations $\partial u / \partial t=F(u, t)+D(t) \Delta u$ where $u=u(\omega, t)$, is a formal analogue of the synchronization of ordinary differential equation (ODE) systems, in the sense explained next. 
Consider interconnected systems $\dot{x}_{i}=f\left(x_{i}, t\right)+\sum_{j \in N(i)} D(t)\left(x_{j}-x_{i}\right)$, where the $i$ th subsystem (or "agent") has state $x_{i}(t)$. An interconnection graph provides the adjacency structure, and the indices in $N(i)$ represent the "neighbors" of the $i$ th subsystem. The matrix $D(t)$ is a diagonal matrix of diffusion strengths. The system synchronizes when the difference between any two states goes to zero exponentially, i.e., $\forall i, j,\left(x_{i}-x_{j}\right)(t) \rightarrow 0$ as $t \rightarrow \infty$. In [2] a sufficient condition for synchronization of such an interconnected system is given.

In the analogy, we think of $u(\omega, \cdot)$ as representing an individual system or agent (the index " $i$ " in the synchronization problem) whose state is described at time $t$ by $u=u(\omega, t)$. (So $u=u(\omega, t)$ plays the role of $x_{i}(t)$. We use " $u$ " to denote the state, instead of $x$, so as to be consistent with standard PDE notations.) Questions of convergence to uniform solutions in reaction diffusion PDEs are also a classical topic of research. We think of convergence to spatially uniform solutions as a sort of "synchronization" of independent "agents", one at each spatial location, and each evolving according to a dynamics specified by an ODE. In that interpretation, our work is related to a large literature on synchronization of discrete groups of agents connected by diffusion, whose interconnections are specified by an undirected graph.

Our methods in this paper, as in [1, 2], are based on contraction theory and matrix measures. They allow one to obtain quantitatively tighter estimates, at least for several PDEs of biological interest, than classical results such as those in $[3,4]$ (comparisons are given in Section 3). The organization of this paper is as follows. In Section 2, after reviewing some of the introductory concepts discussed in [1], we will review previous related works, and then we will state and prove the main result of this work. In Section 3, we will review the biochemical example described in $[5,6]$ and the Goodwin example studied in $[7,8]$, in the current context.

\section{Spatial uniformity of solutions of reaction diffusion PDEs}

\subsection{Preliminaries: logarithmic Lipschitz constants}

In this paper, we focus on conditions based on matrix measures. We recall (see for instance [9] or [10]) that, given a vector norm on Euclidean space $(|\cdot|)$, with its induced matrix norm $\|A\|$, the associated matrix measure $\mu$ is defined as the directional derivative of the matrix norm in the direction of $A$ and evaluated at the identity matrix, that is:

$$
\mu[A]:=\lim _{h \rightarrow 0^{+}} \frac{1}{h}(\|I+h A\|-1) .
$$

As every norm possesses right (and left) Gâteaux-differentials, the limit is known to exist and the convergence is monotonic, see $[11,12]$.

The matrix measure, also known as the "logarithmic norm" of a square matrix $A$, was independently introduced by Germund Dahlquist [11], and Sergei Lozinskii 
[13], in 1959. In 1965, W. A. Coppel [14, page 58] showed that $\mu$ can be used to bound solutions of linear differential equations $\dot{x}=A(t) x$. In 1970, R. H. Martin [15] extended the definition of $\mu$ to functions which satisfy a Lipschitz condition on bounded subsets of a Banach space (see Definition 2) and used this extension to bound solutions of the corresponding differential equations.

For ease of reference, we now borrow some definitions of logarithmic Lipschitz constants which are originally based on the definitions in [16, 17]. For more properties, technical lemmas and proofs see $[1,18]$.

Definition 1. [1, Definition 3] Let $\left(X,\|\cdot\|_{X}\right)$ be a normed space and $f: Y \rightarrow X$ be a function, where $Y \subseteq X$. The least upper bound (lub) Lipschitz constant of $f$ induced by the norm $\|\cdot\|_{X}$, on $Y$, is defined by

$$
L_{Y, X}[f]=\sup _{u \neq v \in Y} \frac{\|f(u)-f(v)\|_{X}}{\|u-v\|_{X}} .
$$

Note that $L_{Y, X}[f]<\infty$ if and only if $f$ is Lipschitz on $Y$.

When identifying a linear operator $f: \mathbb{R}^{n} \rightarrow \mathbb{R}^{n}$ with its matrix representation $A$ with respect to the canonical basis, $L_{Y, X}[A]=\|A\|_{X \rightarrow X}$, where $\|\cdot\|_{X \rightarrow X}$ is the operator norm induced by $\|\cdot\|_{X}$.

Definition 2. [1, Definition 4] Let $\left(X,\|\cdot\|_{X}\right)$ be a normed space and $f: Y \rightarrow X$ be a Lipschitz function. The least upper bound (lub) logarithmic Lipschitz constant of $f$ induced by the norm $\|\cdot\|_{X}$, on $Y \subseteq X$, is defined by

$$
\mu_{Y, X}[f]=\lim _{h \rightarrow 0+} \frac{1}{h}\left(L_{Y, X}[I+h f]-1\right),
$$

or equivalently,

$$
\mu_{Y, X}[f]=\lim _{h \rightarrow 0^{+}} \sup _{u \neq v \in Y} \frac{1}{h}\left(\frac{\|u-v+h(f(u)-f(v))\|_{X}}{\|u-v\|_{X}}-1\right) .
$$

If $X=Y$, we write $\mu_{X}$ instead of $\mu_{X, X}$. Whenever it is clear from the context, we drop the subscript and simply write $\mu$ instead of $\mu_{Y, X}$.

When identifying a linear operator $f: \mathbb{R}^{n} \rightarrow \mathbb{R}^{n}$ with its matrix representation $A$ with respect to the canonical basis, we call $\mu$ a "matrix measure" or a "logarithmic norm".

Notation 1. [1, Definition 2] Let $\left(X,\|\cdot\|_{X}\right)$ be a normed space and $f: Y \rightarrow X$ be a function. Denote $\mu_{Y, X}^{+}$as follows

$$
\mu_{Y, X}^{+}[f]:=\sup _{u \neq v \in Y} \lim _{h \rightarrow 0^{+}} \frac{1}{h}\left(\frac{\|u-v+h(f(u)-f(v))\|_{X}}{\|u-v\|_{X}}-1\right) .
$$

If $X=Y$, we write $\mu_{X}^{+}$instead of $\mu_{X, X}^{+}$. Whenever it is clear from the context, we drop the subscript and simply write $\mu^{+}$instead of $\mu_{Y, X}^{+}$. 
The (lub) logarithmic Lipschitz constant makes sense even if $f$ is not differentiable. However, the constant can be tightly estimated, for differentiable mappings on convex subsets of finite-dimensional spaces, by means of Jacobians, [19]. In this work, $J_{f}$ denotes the Jacobian of $f$.

Notation 2. For any $1 \leq p \leq \infty$, and any nonsingular, diagonal matrix $Q=$ $\operatorname{diag}\left(q_{1}, \ldots, q_{n}\right)$, we introduce a $Q$-weighted norm on $C_{\mathbb{R}^{n}}(\bar{\Omega})$ as follows:

$$
\|v\|_{p, Q}:=\left\|Q\left(\left\|v_{1}\right\|_{p}, \ldots,\left\|v_{n}\right\|_{p}\right)^{T}\right\|_{p}
$$

Since

$$
\|v\|_{p, Q}=\left\{\begin{array}{cc}
\left(\sum_{i}\left|q_{i}\right|^{p}\left\|v_{i}\right\|_{p}^{p}\right)^{\frac{1}{p}} & 1 \leq p<\infty \\
\sup _{i}\left|q_{i}\right|\left\|v_{i}\right\|_{p} & p=\infty,
\end{array}\right.
$$

without loss of generality we will assume $q_{i}>0$ for each $i$. Note that $\|v\|_{p, Q}$ is finite, for any $p, Q$, because each $v_{i}$ is a continuous function on $\bar{\Omega}$ and $\bar{\Omega}$ is a compact subset of $\mathbb{R}^{m}$.

With a slight abuse of notation, we use the same symbol for a norm in $\mathbb{R}^{n}$ :

$$
\|x\|_{p, Q}:=\|Q x\|_{p}
$$

For $\left(X,\|\cdot\|_{p}\right)$, where $\|\cdot\|_{p}$ is the $L^{p}$ norm on $X$, for some $1 \leq p \leq \infty$, we sometimes use the notation " $\mu_{p}$ " instead of $\mu_{X}$ for the least upper bound logarithmic Lipschitz constant or logarithmic norm, and by " $\mu_{p, Q}$ " we denote the least upper bound logarithmic Lipschitz constant or logarithmic norm induced by the weighted $L^{p}$ norm, $\|u\|_{p, Q}:=\|Q u\|_{p}$, where $Q$ is a fixed nonsingular matrix.

Lemma 1. [1, Lemma 4 and Corollary 1] Let $\left(X,\|\cdot\|_{X}\right)$ be a normed space and $G: Y \times[0, \infty) \rightarrow X$ be a Lipschitz function on its first argument, where $Y \subseteq X$. Let $u, v:[0, \infty) \rightarrow Y$ be two solutions of

$$
\frac{d u(t)}{d t}=G_{t}(u(t))
$$

where $G_{t}(u)=G(u, t)$. Then for all $t \in[0, \infty)$,

$$
D^{+}\|(u-v)(t)\|_{X}=\frac{\left((u-v)(t), G_{t}(u(t))-G_{t}(v(t))\right)_{+}}{\|(u-v)(t)\|_{X}^{2}}\|(u-v)(t)\|_{X},
$$

where $D^{+}$denotes the upper right Dini derivative. In addition,

$$
D^{+}\|(u-v)(t)\|_{X} \leq \mu^{+}\left[G_{t}\right]\|(u-v)(t)\|_{X} .
$$

Lemma 2. [1, Corollary 3] Let $\left(X,\|\cdot\|_{X}\right)$ be a normed space and $G: Y \times$ $[0, \infty) \rightarrow X$ be a Lipschitz function on its first argument, where $Y \subseteq X$. Suppose $u, v:[0, \infty) \rightarrow Y$ satisfy

$$
(\dot{u}-\dot{v})(t)=G_{t}(u(t))-G_{t}(v(t)),
$$

where $\dot{u}=\frac{d u}{d t}$ and $G_{t}(u)=G(u, t)$. Let $c:=\sup _{t \in[0, \infty)} \mu_{Y, X}\left[G_{t}\right]$. Then for all $t \in[0, \infty)$,

$$
\|u(t)-v(t)\|_{X} \leq e^{c t}\|u(0)-v(0)\|_{X} .
$$




\subsection{Previous work}

Before we review some previous results and state the main result of this work, we recall the definitions of solutions of reaction diffusion PDEs with Neumann or Dirichlet boundary conditions as follows.

Definition 3. By a solution of the reaction diffusion PDE

$$
\frac{\partial u}{\partial t}(\omega, t)=F(u(\omega, t), t)+D(t) \Delta u(\omega, t)
$$

with

1. Neumann boundary conditions: $\quad \frac{\partial u}{\partial \mathbf{n}}(\xi, t)=0, \quad \forall \xi \in \partial \Omega$; or

2. Dirichlet boundary condition: $u(\xi, t)=0, \quad \forall \xi \in \partial \Omega$,

and with initial condition

$$
u(\omega, 0)=u_{0}(\omega), \quad \omega \in \Omega
$$

on an interval $[0, T)$, where $0<T \leq \infty$, we mean a function $u=\left(u_{1}, \ldots, u_{n}\right)^{T}$, with $u: \bar{\Omega} \times[0, T) \rightarrow V$, such that:

- for each $\omega \in \bar{\Omega}, u(\omega, \cdot)$ is continuously differentiable;

- for each $t \in[0, T), u(\cdot, t)$ is in

1. $\mathbf{Y}_{V}^{(n)}=\left\{v: \bar{\Omega} \rightarrow V, v=\left(v_{1}, \ldots, v_{n}\right)^{T}, v_{i} \in C_{\mathbb{R}}^{2}(\bar{\Omega}), \frac{\partial v_{i}}{\partial \mathbf{n}}(\xi)=0, \forall \xi \in \partial \Omega, \forall i\right\}$ (the superscript $(n)$ is for Neumann); or

2. $\mathbf{Y}_{V}^{(d)}=\left\{v: \bar{\Omega} \rightarrow V, v=\left(v_{1}, \ldots, v_{n}\right)^{T}, v_{i} \in C_{\mathbb{R}}^{2}(\bar{\Omega}), v_{i}(\xi)=0, \forall \xi \in \partial \Omega, \forall i\right\}$, (the superscript $(d)$ is for Dirichlet);

- for each $\omega \in \bar{\Omega}$, and each $t \in[0, T)$, u satisfies the above PDE.

Since we are interested here in estimates relating pairs of solutions, we do not need to deal with well-posedness of the solutions. Our results will refer to solutions that are already assumed to exist and are global.

Remark 1. There are various sufficient conditions that imply global existence of solutions. For example, when the diffusion matrix $D$ is diagonal and the diffusion constants are different, the following extra conditions on $F$ and $V$ guarantee a unique (global) solution of Equation (1) (see [20, Chapter 14] for more details about existence of the solutions):

- The convex set $V$ is a rectangle of the following form

$$
V=\left\{x \in \mathbb{R}^{n}: a_{i} \leq x_{i} \leq b_{i}\right\} \text { for some constants } a_{i} \text { 's and } b_{i} \text { 's. }
$$

- The vector field $F(x, t)$ is twice continuously differentiable on $x$ and continuous on $(x, t)$. 
- The vector field $F$ points strictly into $V$, i.e., for any outward normal vector $\vec{n}$ of $\partial V, F \cdot \vec{n}<0$.

These extra conditions guarantee that $V$ is invariant (which means $u(\omega, 0) \in V$ implies $u(\omega, t) \in V$, for any $t \in[0, T)$ and any $\omega \in \Omega$ ) and Equation (1) has a unique solution.

The $a_{i}$ 's and $b_{i}$ 's could be $\pm \infty$; but finite $a_{i}$ 's and $b_{i}$ 's guarantee global existence of the solutions.

Reaction diffusion PDEs with equal diffusion constants, i.e., $D=a I$ for some $a>0$, can admit any convex invariant set (provided that $F$ points into the convex set). But when the diffusion constants are different, this cannot happen. For example (see [21, page 158]), consider two heat equations $(F=0)$ with constant coefficients $d_{1}$ and $d_{2}$ on the open interval $(0,1)$ and with equal "non-constant" initial conditions. Obviously, the convex set $V=\left\{u=\left(u_{1}, u_{2}\right): u_{1}=u_{2}\right\}$ is an invariant set when $d_{1}=d_{2}$, but it is not an invariant set when $d_{1} \neq d_{2}$.

Note that $F$ and $V$ in the examples that we study in this work, admit invariant rectangles .

More theorems on existence and uniqueness for PDEs such as (1) can be found in standard references, e.g., [22, 23] and [24, 25, 26].

Definition 4. We say that the reaction diffusion PDE (1) is contractive, if for any two solutions $u, v$ of (1), subject to Neumann or Dirichlet boundary conditions, $\|(u-v)(\cdot, t)\| \rightarrow 0$ as $t \rightarrow \infty$.

Definition 5. We say that the reaction diffusion PDE (1) synchronizes, if for any solution $u$ of (1), subject to Neumann or Dirichlet boundary conditions, there exists $\bar{u}(t)$ such that $\|u(\cdot, t)-\bar{u}(t)\| \rightarrow 0$ as $t \rightarrow \infty$, or $\|\nabla u(\cdot, t)\| \rightarrow 0$ as $t \rightarrow \infty$, respectively.

The following theorem, from [1], provides a sufficient condition for contractivity of the reaction diffusion PDE (1) subject to the Neumann boundary condition (2). Then, in Remark 2 below, we show that how contractivity of the reaction diffusion PDE implies synchronization.

Theorem 1. Consider the reaction diffusion PDE (1), defined on $[0, T)$, subject to Neumann boundary conditions (2). Let $c=\sup _{(x, t)} \mu_{p, Q}\left[J_{F}(x, t)\right]$ for some $1 \leq p \leq \infty$, and some positive diagonal matrix $Q$. Then for any two solutions $u, v$ of the PDE and all $t \in[0, T)$ :

$$
\|u(\cdot, t)-v(\cdot, t)\|_{p, Q} \leq e^{c t}\|u(\cdot, 0)-v(\cdot, 0)\|_{p, Q} \cdot
$$

Remark 2. Under the conditions of Theorem 1, if $c=\sup _{(x, t)} \mu_{p, Q}\left[J_{F}(x, t)\right]<0$, any solution $u$ of the $\operatorname{PDE}(1)$ with $u(\omega, 0)=u_{0}(\omega) \in \mathbf{Y}_{V}^{(n)}$, which is defined globally, exponentially converges to the spatially uniform solution $\bar{u}(t)$ which is itself the solution of the following ODE system:

$$
\dot{x}=F(x, t), \quad x(0)=\frac{1}{|\Omega|} \int_{\Omega} u_{0}(\omega) d \omega .
$$


But, note that the condition $c<0$ rules out any interesting non-equilibrium behavior. For instance in the example of Goodwin's oscillatory system, Section 3 below, $c<0$ kills out the oscillation. So we look for a weaker condition than $c<0$, that guarantees spatial uniform convergence (which is a weaker property than contraction) while preserving interesting non-equilibrium behavior, such as oscillations.

Recall, [27], that for any bounded, open subset $\Omega \subset \mathbb{R}^{m}$, there exist a sequence of non-negative eigenvalues $0 \leq \lambda_{1}^{(n)} \leq \lambda_{2}^{(n)} \leq \cdots$ going to $\infty$, (superscript ( $n$ ) for Neumann) and a sequence of corresponding orthonormal eigenfunctions $\phi_{1}^{(n)}, \phi_{2}^{(n)}, \ldots$ (defining a Hilbert basis of $L^{2}(\Omega)$ ) satisfying the following Neumann eigenvalue problem:

$$
\begin{gathered}
-\Delta \phi_{i}^{(n)}=\lambda_{i}^{(n)} \phi_{i}^{(n)} \quad \text { in } \Omega \\
\nabla \phi_{i}^{(n)} \cdot \mathbf{n}=0 \quad \text { on } \partial \Omega .
\end{gathered}
$$

Note that the first eigenvalue is always zero, $\lambda_{1}^{(n)}=0$, and the corresponding eigenfunction is a nonzero constant $\left(\phi_{1}^{(n)}(\omega)=1 / \sqrt{|\Omega|}\right)$.

The following re-phasing of a theorem from [8], provides a sufficient condition on $F$ and $D$ (time invariant diffusion matrix) using the Jacobian matrix of the reaction term and the second Neumann eigenvalue of the Laplacian operator on the given spatial domain to insure the convergence of trajectories, in this case to their space averages in weighted $L^{2}$ norms. The proof in [8] is based on the use of a quadratic Lyapunov function, which is appropriate for Hilbert spaces. We have translated the result to the language of contractions. (Actually, the result in [8] is slightly stronger, in that it allows for non-diagonal diffusion and also non-diagonal weighting matrices $Q$, by substituting these assumptions by a commutativity type of condition, see [28] for more details and a generalization to spatially-varying diffusion.)

Theorem 2. Consider the reaction diffusion system (1) subject to Neumann boundary conditions. Let

$$
c:=\sup _{(x, t) \in V \times[0, \infty)} \mu_{2, Q}\left[J_{F}(x, t)-\lambda_{2}^{(n)} D\right],
$$

where $Q$ is a positive diagonal matrix. Then

$$
\|u(\cdot, t)-\tilde{u}(t)\|_{2, Q} \leq e^{c t}\|u(\cdot, 0)-\tilde{u}(0)\|_{2, Q},
$$

where $\tilde{u}(t)=\frac{1}{|\Omega|} \int_{\Omega} u(\omega, t) d \omega$.

Note that when $c<0$ and $u$ is defined globally, the reaction diffusion system (1) synchronizes.

The following theorem from [2] is an analogous result to Theorem 2 for any norm but restricted to the linear operators $F, F(u, t)=A(t) u$, where for any $t$, $A(t) \in \mathbb{R}^{n \times n}$. 
Theorem 3. Consider the reaction diffusion system (1), for a linear operator F. For a given norm $\|\cdot\|$ in $\mathbb{R}^{n}$, let

$$
c:=\sup _{(x, t) \in V \times[0, \infty)} \mu\left[J_{F}(x, t)-\lambda_{2}^{(n)} D(t)\right]
$$

where $\mu$ is the logarithmic norm induced by $\|\cdot\|$. Then for any $\omega \in \Omega$ and any $t \geq 0$

$$
\|u(\omega, t)-\bar{u}(t)\| \leq \sum_{i \geq 2}\left\|\alpha_{i}(t) \phi_{i}^{(n)}(\omega)\right\| \leq e^{c t} \sum_{i \geq 2}\left\|\alpha_{i}(0) \phi_{i}^{(n)}(\omega)\right\|,
$$

where $\bar{u}(t)$ is the solution of the system (10) with initial condition $u_{0}(\omega)=u(\omega, 0)$, and $\alpha_{i}(t)=\int_{\Omega} u(\omega, t) \phi_{i}^{(n)}(\omega) d \omega$. In particular, when $c<0$,

$$
\|u(\omega, t)-\bar{u}(t)\| \rightarrow 0 \quad \text { exponentially, as } t \rightarrow \infty .
$$

\subsection{Main results}

In what follows, we first present some conditions, analogous to the conditions in Theorem 1, which guarantee contractivity of the solutions of a reaction diffusion PDE with Dirichlet boundary conditions. Then, we show that how contractivity of the reaction diffusion PDE with Dirichlet boundary conditions implies spatial uniformity for the asymptotic behavior of the solutions of a reaction diffusion PDE with Neumann boundary conditions. For non-Euclidean norms we only provide results in one dimensional space, and the general problem remains open.

Recall, [27], that for any bounded, open subset $\Omega \subset \mathbb{R}^{m}$, there exists a sequence of positive eigenvalues $0<\lambda_{1}^{(d)} \leq \lambda_{2}^{(d)} \leq \cdots$ going to $\infty$ (superscript $(d)$ for Dirichlet), and a sequence of corresponding orthonormal eigenfunctions $\phi_{1}^{(d)}, \phi_{2}^{(d)}, \ldots$ (defining a Hilbert basis of $L^{2}(\Omega)$ ) satisfying the following Dirichlet eigenvalue problem:

$$
\begin{array}{cc}
-\Delta \phi_{i}^{(d)}=\lambda_{i}^{(d)} \phi_{i}^{(d)} & \text { in } \Omega \\
\phi_{i}^{(d)}=0 & \text { on } \partial \Omega .
\end{array}
$$

Let us assume that $\Omega$ is a connected open set. Then the first eigenvalue $\lambda_{1}^{(d)}$ is simple and the first eigenfunction $\phi_{1}^{(d)}$ has a constant sign on $\Omega$. Without loss of generality, $\phi_{1}^{(d)}$ can be assumed to be everywhere positive on $\Omega$.

We next prove a result analogous to Theorem 1 (restricted to $p=1$ ), for reaction diffusion PDE

$$
\begin{gathered}
\frac{\partial u_{1}}{\partial t}(\omega, t)=F_{1}(u(\omega, t), \omega, t)+d_{1}(t) \frac{\partial^{2} u_{1}}{\partial \omega^{2}}(\omega, t) \\
\vdots \\
\frac{\partial u_{n}}{\partial t}(\omega, t)=F_{n}(u(\omega, t), \omega, t)+d_{n}(t) \frac{\partial^{2} u_{n}}{\partial \omega^{2}}(\omega, t),
\end{gathered}
$$


on $\Omega=(0, L)$, and subject to Dirichlet boundary conditions:

$$
u_{i}(0, t)=u_{i}(L, t)=0, \quad \forall i=1, \ldots, n .
$$

Note that in (13), we let $F$ depend on $\omega$. In fact, $F$ is defined on $V \times \Omega \times[0, \infty)$.

Theorem 4. Consider the reaction diffusion PDE (13) subject to Dirichlet boundary conditions (14). Let

$$
c=\sup _{(\omega, t)} \sup _{x} \mu_{1, Q}\left[J_{F}(x, \omega, t)-\pi^{2} / L^{2} D(t)\right],
$$

and let $u(\omega, t)$ and $v(\omega, t)$ be two solutions of (13) and (14). Then

$$
\|(u-v)(\cdot, t)\|_{1, \phi, Q} \leq e^{c t}\|(u-v)(\cdot, 0)\|_{1, \phi, Q},
$$

where $\|u\|_{1, \phi, Q}=\|\phi u\|_{1, Q}$ and $\phi=\sin (\pi \omega / L)$. Note that for $\Omega=(0, L)$, the first Dirichlet eigenvalue is $\pi^{2} / L^{2}$ and a corresponding eigenfunction is $\sin (\pi \omega / L)$.

To prove Theorem 4, we need the following lemmas:

Lemma 3. Under the conditions of Theorem 4 and for any fixed $t$, let $\mathcal{A}(t)$ denote an $n \times n$ diagonal matrix of operators on $\mathbf{Y}_{V}^{(d)}$ with operators $d_{i}(t) \frac{\partial^{2}}{\partial \omega^{2}}$ on the diagonal. Let $\Lambda^{(d)}(t)$ denote an $n \times n$ diagonal matrix of operators on $\mathbf{Y}_{V}^{(d)}$ with operators $\Lambda_{i}^{(d)}(t)$ on the diagonal which are defined as follows:

$$
\Lambda_{i}^{(d)}(t)(\psi)(\omega):=\lambda_{1}^{(d)} d_{i}(t) \psi_{i}(\omega) .
$$

Then

$$
\mu_{1, \phi, Q}^{+}\left[\mathcal{A}+\Lambda^{(d)}\right]=0,
$$

where $\mu_{1, \phi, Q}^{+}$is induced by $\|\cdot\|_{1, \phi, Q}$.

See the Appendix for a proof.

Lemma 4. Let $G: \mathbb{R}^{n} \times \Omega \rightarrow \mathbb{R}^{n}$ be Lipschitz with respect to the first argument. Define $\hat{G}: \mathbf{Y}_{V}^{(d)} \rightarrow \mathbb{R}^{n}$ as follows:

$$
\hat{G}(u)(\omega):=G(u(\omega), \omega) .
$$

Then,

$$
\mu_{p, \phi, Q}^{+}[\hat{G}] \leq \sup _{\omega \in \Omega} \mu_{p, Q}[G(\cdot, \omega)] .
$$

In addition, if $G$ is continuously differentiable with respect to $x$ then

$$
\mu_{p, \phi, Q}^{+}[\hat{G}] \leq \sup _{\omega \in \Omega} \mu_{p, Q}[G(\cdot, \omega)]=\sup _{\omega \in \Omega} \sup _{x} \mu_{p, Q}\left[J_{G}(x, \omega)\right] .
$$

Note that although we will need this lemma only for $p=1$, the result of this lemma is true for any $1 \leq p \leq \infty$. See the Appendix for a proof.

Proof of Theorem 4. Suppose that $u$ is a solution of Equation (13). Define $\hat{u}$, $\mathcal{H}_{t}$, and $\mathcal{A}$ as follows: 
- $\hat{u}:[0, T) \rightarrow \mathbf{Y}_{V}^{(d)}, \quad \hat{u}(t)(\omega):=u(\omega, t)$

- $\mathcal{H}_{t}: \mathbf{Y}_{V}^{(d)} \rightarrow \mathbb{R}^{n}, \quad \mathcal{H}_{t}(\psi)(\omega):=F(\psi(\omega), \omega, t), \quad \forall \psi \in \mathbf{Y}_{V}^{(d)}, \quad \forall \omega \in \Omega$

- $\mathcal{A}$ is as defined in Lemma 3.

Then by the definition,

$$
\frac{d \hat{u}}{d t}(t)=\left(\mathcal{H}_{t}+\mathcal{A}\right)(\hat{u}(t))
$$

Suppose $u$ and $v$ are two solutions of Equation (13). By Lemma 1 and Equation (18) we have:

$$
D^{+}\|(\hat{u}-\hat{v})(t)\|_{1, \phi, Q} \leq \mu_{1, \phi, Q}^{+}\left[\mathcal{H}_{t}+\mathcal{A}\right]\|(\hat{u}-\hat{v})(t)\|_{1, \phi, Q} .
$$

Let $\Lambda^{(d)}$ be as in Lemma 3. By subadditivity property of $\mu^{+}$(see [1, Proposition 2]), Lemma 3 and Lemma 4, we have:

$$
\begin{aligned}
\mu_{1, \phi, Q}^{+}\left[\mathcal{H}_{t}+\mathcal{A}\right] & \leq \mu_{1, \phi, Q}^{+}\left[\mathcal{H}_{t}-\Lambda^{(d)}\right]+\mu_{1, \phi, Q}^{+}\left[\mathcal{A}+\Lambda^{(d)}\right] \\
& \leq \mu_{1, \phi, Q}^{+}\left[\mathcal{H}_{t}-\Lambda^{(d)}\right] \\
& \leq \sup _{\omega \in \Omega} \sup _{x \in V} \mu_{1, Q}\left[J_{F}(x, \omega, t)-\lambda_{1}^{(d)} D(t)\right] \\
& \leq \sup _{t \in[0, T)} \sup _{\omega \in \Omega} \sup _{x \in V} \mu_{1, Q}\left[J_{F}(x, \omega, t)-\lambda_{1}^{(d)} D(t)\right] \\
& =c .
\end{aligned}
$$

By (19), (20), and Lemma 2, we get:

$$
\|(\hat{u}-\hat{v})(t)\|_{1, \phi, Q} \leq e^{c t}\|(\hat{u}-\hat{v})(0)\|_{1, \phi, Q} .
$$

In terms of the PDE (13), this last estimate can be equivalently written as:

$$
\|(u-v)(\cdot, t)\|_{1, \phi, Q} \leq e^{c t}\|(u-v)(\cdot, 0)\|_{1, \phi, Q} .
$$

Note that unlike in Neumann boundary problems, one cannot conclude synchronization from contraction in the Dirichlet boundary problems unless for any $t$ and $\omega, F(0, \omega, t)=0$ :

Corollary 1. Under the conditions of Theorem 4, if for any $(\omega, t), F(0, \omega, t)=0$, then $v=0$ is a uniformly spatial solution of Equations (1) and (3), and therefore, for any solution $u$ of Equations (1) and (3),

$$
\|u(\cdot, t)\|_{1, \phi, Q} \leq e^{c t}\|u(\cdot, 0)\|_{1, \phi, Q},
$$

Hence, when $c<0$, the PDE system synchronizes. 
The following theorem is our main result. It provides a sufficient condition for synchronization of reaction diffusion systems subject to the Neumann boundary condition restricted to one dimensional space and $p=1$. The proof is based on Theorem 4.

Theorem 5. Let $u(\omega, t)$ be a solution of

$$
\begin{aligned}
& \frac{\partial u}{\partial t}(\omega, t)=F(u(\omega, t), \omega, t)+D(t) \frac{\partial^{2} u}{\partial \omega^{2}}(\omega, t) \quad \text { in } \Omega=(0, L) \\
& \frac{\partial u}{\partial \omega}(0, t)=\frac{\partial u}{\partial \omega}(L, t)=0,
\end{aligned}
$$

defined for all $t \in[0, T)$ for some $0<T \leq \infty$. In addition, assume that $u(\cdot, t) \in$ $C^{3}(\bar{\Omega})$, for all $t \in[0, T)$. Let

$$
c=\sup _{\omega \in \Omega} \sup _{t \in[0, T)} \sup _{x \in V} \mu_{1, Q}\left[J_{F}(x, \omega, t)-\pi^{2} / L^{2} D(t)\right] .
$$

Then for all $t \in[0, T)$ :

$$
\left\|\frac{\partial u}{\partial \omega}(\cdot, t)\right\|_{1, \phi, Q} \leq e^{c t}\left\|\frac{\partial u}{\partial \omega}(\cdot, 0)\right\|_{1, \phi, Q},
$$

where

$$
\|\cdot\|_{1, \phi, Q}:=\|\sin (\pi \omega / L)(\cdot)\|_{1, Q} .
$$

The significance of Theorem 5 lies in the fact that $\sin (\pi \omega / L)$ is nonzero everywhere in the domain (except at the boundary). In that sense, we have exponential convergence to uniform solutions in a weighted $L^{1}$ norm, the weights being specified in $V$ by the matrix $Q$ and in space by the function $\sin (\pi \omega / L)$.

Proof. Suppose that $u$ is a solution of Equation (21), and let $v=\frac{\partial u}{\partial \omega}$. Then by taking $\frac{\partial}{\partial \omega}$ on both sides of Equation (21), we get the following PDE:

$$
\frac{\partial v}{\partial t}=J_{F}(u(\omega), t) v+D(t) \frac{\partial^{2} v}{\partial \omega^{2}}
$$

subject to Dirichlet boundary condition: $v(0)=v(L)=0$. Note that since we assume that $u(\cdot, t) \in C^{3}(\bar{\Omega})$, for all $t \in[0, T), \partial^{2} v / \partial \omega^{2}$ makes sense. Therefore, by Equation (23) and Corollary 1,

$$
\|v(\cdot, t)\|_{1, \phi, Q} \leq e^{c t}\|v(\cdot, 0)\|_{1, \phi, Q},
$$

where $c=\sup _{t \in[0, T)} \sup _{\omega \in \Omega} \sup _{x \in V} \mu_{1, Q}\left[J_{F}(x, \omega, t)-\pi^{2} / L^{2} D(t)\right]$.

Another proof of Theorem 5, using the method of discretization is given in the Appendix.

Remark 3. In the case of $\Omega=(0, L), \lambda_{1}^{(d)}=\lambda_{2}^{(n)}$. Therefore, one can state the conditions of Theorem 5 in terms of the second Neumann eigenvalue instead of the first Dirichlet eigenvalue. 


\section{Examples}

Example 1. In [1], we studied the following system of PDEs on $\Omega=(0,1)$ with Neumann boundary conditions, which models a simple biochemical binding/unbinding system:

$$
\begin{aligned}
& \frac{\partial x}{\partial t}=z(t)-\delta x+k_{1} y-k_{2}\left(S_{Y}-y\right) x+d_{1} \Delta x \\
& \frac{\partial y}{\partial t}=-k_{1} y+k_{2}\left(S_{Y}-y\right) x+d_{2} \Delta y,
\end{aligned}
$$

where $x=x(\omega, t), y=y(\omega, t), z(t)$ is a positive function, $\delta, k_{1}, k_{2}, S_{Y}$ are positive constants, and $d_{1}, d_{2}$ are non-negative diffusion constants. We constructed there a positive diagonal matrix $Q$ such that

$$
c:=\sup _{(x, y) \in V} \mu_{1, Q}\left[J_{F}(x, y)\right]<0,
$$

where the vector field $F=\left(z(t)-\delta x+k_{1} y-k_{2}\left(S_{Y}-y\right) x,-k_{1} y+k_{2}\left(S_{Y}-y\right) x\right)^{T}$, with the convex domain $V=[0, \infty) \times\left[0, S_{Y}\right]$.

Therefore, by Remark 2, we conclude that any solution of (24) converges to a uniform solution with at least rate $c$.

Next, using Theorem 5, we show that any solution of (24) converges to a uniform solution at a better rate than $c$.

By subadditivity of $\mu$, we have:

$$
\sup _{(x, y) \in V} \mu_{1, Q}\left[J_{F}(x, y)-\pi^{2} D\right] \leq \sup _{(x, y) \in V} \mu_{1, Q}\left[J_{F}(x, y)\right]-\pi^{2} d,
$$

where $d=\min \left\{d_{1}, d_{2}\right\}$. Therefore,

$$
c_{0}:=\sup _{(x, y) \in V} \mu_{1, Q}\left[J_{F}(x, y)-\pi^{2} D\right]<c<0 .
$$

Hence, by Theorem 5, for any solution $u=(x, y)^{T}$ of $(24)$ :

$$
\left\|\frac{\partial u}{\partial \omega}(\cdot, t)\right\|_{1, \phi, Q} \leq e^{c_{0} t}\left\|\frac{\partial u}{\partial \omega}(\cdot, 0)\right\|_{1, \phi, Q},
$$

where in this example $\phi(\omega)=\sin (\pi \omega)$, since $\Omega=(0,1)$.

Also, in [2], we showed that for any non-negative $\lambda$ and any positive diagonal matrix $D=\operatorname{diag}\left(d_{1}, d_{2}\right)$,

$$
c:=\sup _{(x, y)} \mu_{2, Q}\left[J_{F}(x, y)-\lambda D\right] \geq 0,
$$

and therefore, we cannot apply the existing results based on $L^{2}$ norms (e.g. [8]) to show the asymptotically uniform behavior of the solutions of (24).

Figure 1 indicates two different solutions of the biochemical model, Equation (24), namely $\left(x_{1}, y_{1}\right)^{T}$ and $\left(x_{2}, y_{2}\right)^{T}$ on $\Omega=(0,2)$, with the following initial conditions,

$$
(\cos (\pi x), 0.05(1-\cos (2 \pi x)))^{T} \in \mathbf{Y}_{V}^{(n)}, \quad(2-\cos (2 \pi x), 0.1 \cos (\pi x))^{T} \in \mathbf{Y}_{V}^{(n)},
$$



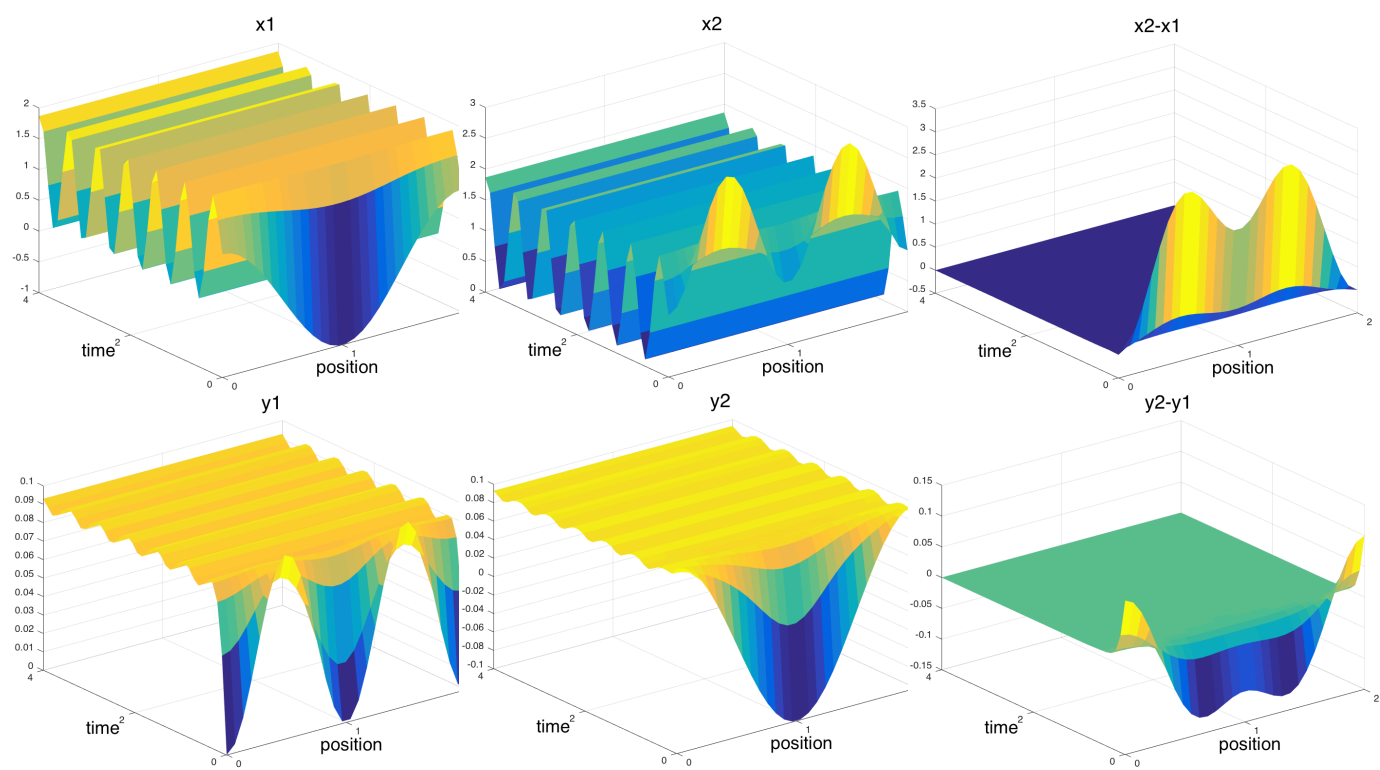

Figure 1: Two solutions $\left(x_{1}, y_{1}\right)^{T}$ and $\left(x_{2}, y_{2}\right)^{T}$ of Equation (24) and their differences $\left(x_{2}-x_{1}, y_{2}-y_{1}\right)^{T}$.

and for a periodic input $z$, namely $z(t)=20(1+\sin (10 t))$, using the following set of parameters:

$$
\delta=20, k_{1}=0.5, k_{2}=5, S_{Y}=0.1, d_{1}=0.001, d_{2}=0.1, M=10 .
$$

Figure 1 also shows how the difference between these two solutions goes to zero as expected.

Remark 4. In the biochemical example, if we assume that $z(t)$ is upper bounded by $\mathcal{M}>0$, we can show that the following closed rectangle

$$
V=[0, M] \times\left[0, S_{Y}\right], \quad \text { with } \quad M>\frac{\mathcal{M}+k_{1} S_{Y}}{\delta},
$$

is forward invariant and hence the reaction diffusion system has a global solution.

Since $V$ is a rectangle, it suffices to show that $F \cdot \vec{n}<0$, for any outward normal vector $\vec{n}$ of the boundary of $V$. For example, as shown in Figure $2, \vec{n}=(-1,0)$ is the normal vector of $\left\{x=0,0 \leq y \leq S_{Y}\right\}$, and

$$
F \cdot \vec{n}=-z(t)-k_{1} y \leq 0, \quad \text { because both } z(t) \text { and } y \text { are non-negative. }
$$

Similarly, one can show that $F \cdot \vec{n}<0$, for the other normal vectors.

Example 2. In 1965, Brian Goodwin proposed a differential equation model, that describes a generic model of an oscillating autoregulatory gene, and studied its oscillatory behavior [29]. In [2], we studied the following systems of ODEs 


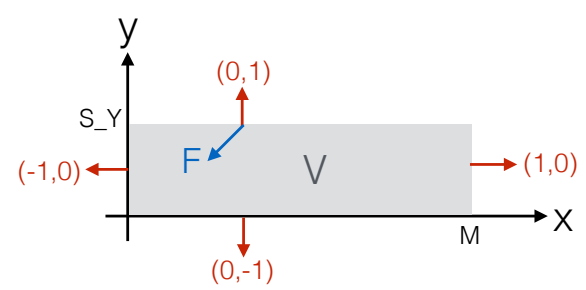

Figure 2: $V=[0, M] \times\left[0, S_{Y}\right]$ is an invariant set for Equation (24).

which is a variant of Goodwin's model [30]:

$$
\begin{aligned}
& \dot{x}=\frac{a}{k+z}-b x \\
& \dot{y}=\alpha x-\beta y \\
& \dot{z}=\gamma y-\frac{\delta z}{k_{M}+z} .
\end{aligned}
$$

Here, we assume a continuous model where species diffuse in space, namely in $\Omega=(0,1)$. This example has been studied in [8]. The following system of PDEs, subject to Neumann boundary conditions, describes the evolution of $x(\omega, t), y(\omega, t)$, and $z(\omega, t)$ where $(\omega, t) \in(0,1) \times[0, \infty)$ :

$$
\begin{aligned}
& \frac{\partial x}{\partial t}=\frac{a}{k+z}-b x+d_{1} \Delta x \\
& \frac{\partial y}{\partial t}=\alpha x-\beta y+d_{2} \Delta y \\
& \frac{\partial z}{\partial t}=\gamma y-\frac{\delta z}{k_{M}+z}+d_{3} \Delta z
\end{aligned}
$$

where $F=\left(\frac{a}{k+z}-b x, \alpha x-\beta y, \gamma y-\frac{\delta z}{k_{M}+z}\right)^{T}$, and $(x, y, z)^{T} \in V=[0, \infty)^{3}$ (since $x, y$, and $z$ could be the concentrations of some chemicals, we assume that they are all non-negative).

Note that $V=[0, \infty)^{3}$ is a convex set and one can show that it is an invariant set by showing that $F \cdot \vec{n}<0$ where $\vec{n}$ is the outward normal vector on $\partial V$.

Figure 3 provides plots of solutions $x, y$, and $z$ of (25), using the following parameter values from the textbook [7]:

$$
a=150, k=1, b=\alpha=\beta=\gamma=0.2, \delta=15, K_{M}=1,
$$

and with the following initial condition,

$$
(100(\cos (2 \pi x)+2), 50(\cos (5 \pi x)+3), 100(\cos (6 \pi x)+1))^{T} \in \mathbf{Y}_{V}^{(n)},
$$

which results in oscillation when there is no diffusion $\left(d_{1}=d_{2}=d_{3}=0\right)$.

A simple calculation shows that for the parameters given in Equation (26), the weighted matrix $Q=\operatorname{diag}(1,12,11)$, and diffusion constants $2.2 / \pi^{2}<d_{1}$, and $d_{2}=d_{3}=0$, the following inequality holds

$$
\sup _{w=(x, y, z)^{T} \in V} \mu_{1, Q}\left[J_{F}(w)-\pi^{2} D\right]<0 .
$$



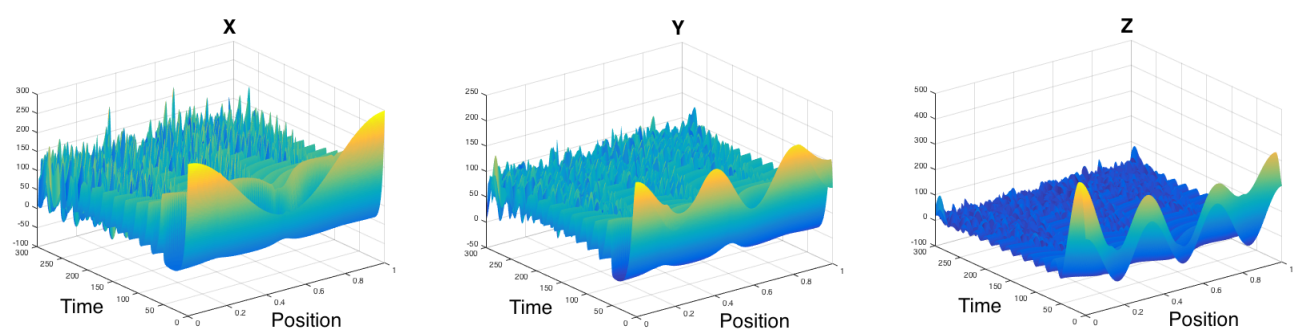

Figure 3: Goodwin oscillator, no diffusion (parameters as in Equation (26))
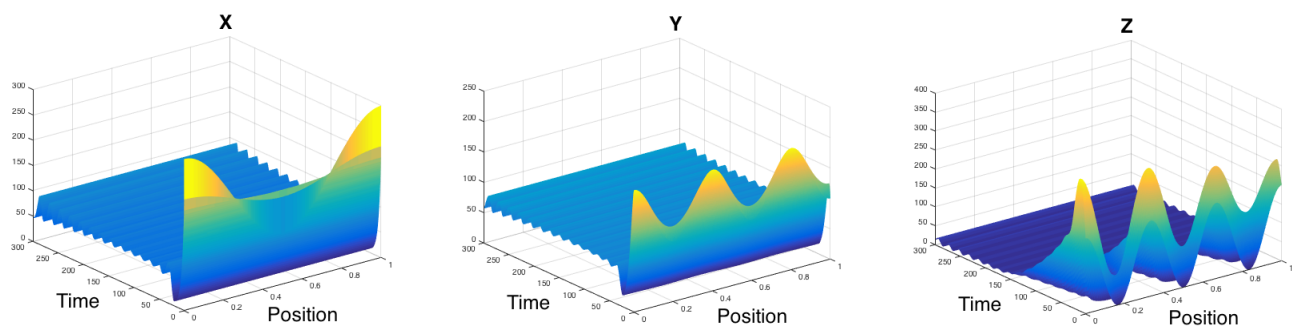

Figure 4: Goodwin oscillator, $x$ diffuses (parameters as in Equation (26))

Applying Theorem 5, we conclude that for $2.2 / \pi^{2}<d_{1}$ and $d_{2}=d_{3}=0$, Equation (25) synchronizes, meaning that solutions tend to uniform solutions. Note that to have synchronization, $2.2 / \pi^{2}$ is not a sharp lower bound for $d_{1}$, i.e., the system would synchronize even for smaller values of $d_{1}$.

Figure 4 shows the spatially uniformity of the solutions of (25), for the same parameter values and initial conditions as in Figure 3, when $2.2 / \pi^{2}<d_{1}$, here $d_{1}=0.3$, and $d_{2}=d_{3}=0$.

In what follows, we compare our result with the results in [8] and [3] using the Goodwin example.

Considering Equation (25), the following sufficient condition in ([8], Equation 55) is given by Arcak for synchronization:

$$
\frac{\alpha \gamma a}{k\left(b+\lambda d_{1}\right)\left(\beta+\lambda d_{2}\right) \lambda d_{3}}<4
$$

where $\lambda=\pi^{2}$. Note that when $d_{3}=0$, one cannot apply (27) directly to get synchronization.

In ([3], Equation 3), Othmer provides a sufficient condition for uniform behavior of the solutions of the reaction diffusion PDE $(1)$ on $(0, L)$, subject to Neumann boundary conditions:

$$
\sup _{w}\left\|J_{F}(w)\right\|<\pi^{2} / L^{2} \min _{i} d_{i}
$$

In Goodwin's example (25), $\sup _{w}\left\|J_{F}(w)\right\|$ is positive and finite (the sup is taken at $z=0$ ), and $\min _{i} d_{i}=0$, hence (28) doesn't hold and this condition is not applicable for this example. 
Note that in [4], Conway et al. independently provided condition (28) for spatial homogeneity in $L^{2}$. In fact, they showed spatial homogeneity in $L^{2}$ for a more general class of reaction diffusion systems.

\section{Open problems}

Although synchronization of the PDE system (1) in weighted $L^{2}$ norms is a wellunderstood problem, the problem is still open for general norms. In Theorem 5 we only showed the result for weighted $L^{1}$ norm and restricted to one dimensional spaces.

\section{Acknowledgements}

Work supported in part by grants AFOSR FA9550-14-1-0060 and ONR 5710003367.

\section{$5 \quad$ Appendix}

\section{Proof of Lemma 3}

To prove Lemma 3, we need the following lemma:

Lemma 5. Pick any $u:(0, L) \rightarrow \mathbb{R}$ such that $\Delta u=\frac{\partial^{2} u}{\partial \omega^{2}}$ is defined on $(0, L)$. Then, there exists a set $I \subset(0, L)$ such that:

- $\mu(I)=0$, where $\mu$ denotes Lebesgue measure; and

- $\Delta|u|$ is defined on $(0, L) \backslash I$.

In fact, we may take $I=\left\{\omega \in(0, L): u(\omega)=0, \frac{\partial u}{\partial \omega}(\omega) \neq 0\right\}$.

Proof. By continuity of $u$, for any point $\omega^{*} \in I$, there is an interval $I^{*}$, such that $\omega^{*} \subset I^{*} \subset I$ and $u$ is either increasing or decreasing in that interval. Therefore, $I \cap I^{*}=\left\{\omega^{*}\right\}$ and thus each $\omega^{*} \in I$ is isolated. Hence, $I$ is countable and of measure zero.

If $u>0$ or $<0$, then it is trivial that $\Delta|u|=|\Delta u|$. Suppose that $u\left(\omega^{*}\right)=0$ and $\frac{\partial u}{\partial \omega}\left(\omega^{*}\right)=0$. Then $u(\omega)=\left(\omega-\omega^{*}\right)^{2} v(\omega)$ for some function $v$. Then

$$
\Delta u(\omega)=2 v(\omega)+\left(\omega-\omega^{*}\right)^{2} \Delta v(\omega)+4\left(\omega-\omega^{*}\right) \frac{\partial v}{\partial \omega}(\omega)
$$

On the other hand,

$$
\frac{\partial}{\partial \omega}|u|(\omega)=\left\{\begin{array}{cc}
\left|2\left(\omega-\omega^{*}\right) v(\omega)+\left(\omega-\omega^{*}\right)^{2} \frac{\partial v}{\partial \omega}(\omega)\right| & v(\omega) \neq 0 \\
0 & v(\omega)=0 .
\end{array}\right.
$$


Therefore,

$$
\Delta|u|(\omega)= \begin{cases}\left|2 v(\omega)+\left(\omega-\omega^{*}\right)^{2} \Delta v(\omega)+4\left(\omega-\omega^{*}\right) \frac{\partial v}{\partial \omega}(\omega)\right| & v(\omega) \neq 0 \\ \lim _{\nu \rightarrow \omega} \frac{1}{\nu-\omega}\left|2\left(\omega-\omega^{*}\right) v(\omega)+\left(\omega-\omega^{*}\right)^{2} \frac{\partial v}{\partial \omega}(\omega)\right| & v(\omega)=0 .\end{cases}
$$

Hence, by computing (29) and (30) at $\omega=\omega^{*}$, we get:

$$
\Delta|u|\left(\omega^{*}\right)=\left|2 v\left(\omega^{*}\right)\right|=\left|\Delta u\left(\omega^{*}\right)\right| .
$$

Proof of Lemma 3. By definition of $\mu_{1, \phi, Q}^{+}$we have:

$$
\mu_{1, \phi, Q}^{+}\left[\mathcal{A}+\Lambda^{(d)}\right]=\sup _{u \in \mathbf{Y}_{V}^{(d)}} \lim _{h \rightarrow 0^{+}} \frac{1}{h}\left\{\frac{\sum_{i} q_{i} \int_{\Omega} \phi(\omega)\left|u_{i}+h d_{i}(t)\left(\Delta+\lambda_{1}^{(d)}\right) u_{i}(\omega)\right| d \omega}{\sum_{i} q_{i} \int_{\Omega} \phi(\omega)\left|u_{i}(\omega)\right| d \omega}-1\right\}
$$

it is enough to show that for a fixed $u \neq 0 \in \mathbf{Y}_{V}^{(d)}$ and a fixed $i=1, \ldots, n$, and fixed $t$ :

$$
\lim _{h \rightarrow 0^{+}} \frac{1}{h}\left\{\int_{\Omega} \phi(\omega)\left|u_{i}(\omega)+h d_{i}(t)\left(\Delta+\lambda_{1}^{(d)}\right) u_{i}(\omega)\right| d \omega-\int_{\Omega} \phi(\omega)\left|u_{i}\right| d \omega\right\}=0 .
$$

Or equivalently, after dividing by $d_{i}(t) \int_{\Omega} \phi(\omega)\left|u_{i}\right| d \omega$, (note that if $d_{i}(t)=0$, then the left hand side of (31) is zero, so we assume that $\left.d_{i}(t) \neq 0\right)$ and renaming $d_{i}(t) h$ as $h$, and dropping $i$, we need to show that:

$$
\lim _{h \rightarrow 0^{+}} \frac{1}{h}\left\{\frac{\int_{\Omega} \phi(\omega)\left|u(\omega)+h\left(\Delta+\lambda_{1}^{(d)}\right) u(\omega)\right| d \omega}{\int_{\Omega} \phi(\omega)|u| d \omega}-1\right\}=0 .
$$

Let $I$ be as in Lemma 5: the set of points of $\Omega=(0, L)$ such that for any $\omega \in I$, $u(\omega)=0$ and $\frac{\partial u}{\partial \omega}(\omega) \neq 0$. To show that Equation (32) holds, we add and subtract $\phi(\omega)\left(|u|+h \Delta|u|+\lambda_{1}^{(d)}|u|\right)$ in the integral of the numerator of the left hand side of (32), and get:

$$
\begin{aligned}
& \lim _{h \rightarrow 0^{+}} \frac{1}{h}\left\{\frac{\int_{\Omega} \phi(\omega)\left|u+h\left(\Delta+\lambda_{1}^{(d)}\right) u\right| d \omega}{\int_{\Omega} \phi(\omega)|u| d \omega}-1\right\} \\
& =\lim _{h \rightarrow 0^{+}} \frac{1}{h}\left\{\frac{\int_{\Omega} \phi(\omega)\left(|u|+h\left(\Delta+\lambda_{1}^{(d)}\right)|u|\right) d \omega}{\int_{\Omega} \phi(\omega)|u| d \omega}-1\right\} \\
& +\lim _{h \rightarrow 0^{+}} \frac{1}{h}\left\{\frac{\int_{\Omega} \phi(\omega)\left(\left|u+h\left(\Delta+\lambda_{1}^{(d)}\right) u\right|-|u|-h\left(\Delta+\lambda_{1}^{(d)}\right)|u|\right) d \omega}{\int_{\Omega} \phi(\omega)|u| d \omega}\right\} .
\end{aligned}
$$


First, we show that the first term of the right hand side of (33) is 0 . By the Divergence Theorem and Dirichlet boundary conditions, we have (recall that $\left.\phi=\phi_{1}^{(d)}\right)$ :

$$
\begin{aligned}
\int_{\Omega} \phi_{1}^{(d)} \Delta|u| & =\int_{\partial \Omega} \phi_{1}^{(d)} \nabla|u| \cdot \mathbf{n}-\int_{\Omega} \nabla|u| \cdot \nabla \phi_{1}^{(d)} \quad\left(\phi_{1}=0 \text { on } \partial \Omega\right) \\
& =-\int_{\partial \Omega} \nabla \phi_{1}^{(d)}|u| \cdot \mathbf{n}+\int_{\Omega}|u| \Delta \phi_{1}^{(d)} \quad(u=0 \text { on } \partial \Omega) \\
& =\int_{\Omega}|u| \Delta \phi_{1}^{(d)} \\
& =-\int_{\Omega}|u| \lambda_{1}^{(d)} \phi_{1}^{(d)} .
\end{aligned}
$$

Therefore,

$$
\int_{\Omega} \phi(\omega)\left(\lambda_{1}^{(d)}+\Delta\right)|u|(\omega) d \omega=0
$$

and so:

$$
\lim _{h \rightarrow 0^{+}} \frac{1}{h}\left\{\frac{\int_{\Omega} \phi(\omega)\left(|u|+h\left(\Delta+\lambda_{1}^{(d)}\right)|u|\right) d \omega}{\int_{\Omega} \phi(\omega)|u| d \omega}-1\right\}=0 .
$$

Next, we show that the second term of the right hand side of $(33)$ is 0 :

$$
\lim _{h \rightarrow 0^{+}} \frac{1}{h}\left\{\frac{\int_{\Omega} \phi(\omega)\left(\left|u+h\left(\Delta+\lambda_{1}^{(d)}\right) u\right|-|u|-h\left(\Delta+\lambda_{1}^{(d)}\right)|u|\right) d \omega}{\int_{\Omega} \phi(\omega)|u| d \omega}\right\}=0 .
$$

In this part, we drop the superscript $(d)$ for the ease of notation: $\lambda_{1}=\lambda_{1}^{(d)}$. For a fixed $u \in \mathbf{Y}_{V}^{(d)}$, we define $F_{h}$, for any $h>0$, as follows:

$$
F_{h}(\omega):=\frac{1}{h}\left\{\phi(\omega)\left(\left|u+h\left(\Delta+\lambda_{1}\right) u\right|-|u|-h\left(\Delta+\lambda_{1}\right)|u|\right)(\omega)\right\} .
$$

1. First, we will show that there exists $M>0$ such that for all $h$ positive, $\left|F_{h}\right|<M$ almost everywhere:

We study $F_{h}$, for any $h>0$, on the following possible subsets of $W:=\Omega \backslash I$ :

- $W_{1}:=\left\{\omega: u(\omega)>0,\left(\Delta+\lambda_{1}\right) u(\omega) \geq 0\right\}$. By definition,

$$
F_{h}(\omega)=\frac{\phi(\omega)}{h}\left(u+h\left(\Delta+\lambda_{1}\right) u-u-h\left(\Delta+\lambda_{1}\right) u\right)(\omega)=0 .
$$

- $W_{2}:=\left\{\omega: u(\omega)>0,\left(\Delta+\lambda_{1}\right) u(\omega)<0, u>\left|\left(\Delta+\lambda_{1}\right) u\right| h\right\}$. By definition,

$$
F_{h}(\omega)=\frac{\phi(\omega)}{h}\left(u+h\left(\Delta+\lambda_{1}\right) u-u-h\left(\Delta+\lambda_{1}\right) u\right)(\omega)=0 .
$$


- $W_{3}=\left\{\omega: u(\omega)>0,\left(\Delta+\lambda_{1}\right) u(\omega)<0, u<\left|\left(\Delta+\lambda_{1}\right) u\right| h\right\}$. By definition,

$$
F_{h}(\omega)=\frac{\phi(\omega)}{h}\left(-u-h\left(\Delta+\lambda_{1}\right) u-u-h\left(\Delta+\lambda_{1}\right) u\right)(\omega) .
$$

Using the triangle inequality and the assumption $u<\left|\left(\Delta+\lambda_{1}\right) u\right| h$, we get:

$$
\begin{aligned}
\left|F_{h}\right| & <\frac{2}{h} \max _{\Omega}|\phi|\left(|u|+h\left|\left(\Delta+\lambda_{1}\right) u\right|\right) \\
& <4 \max _{\Omega}|\phi|\left|\left(\Delta+\lambda_{1}\right) u\right| \\
& \leq 4 \max _{\Omega}|\phi|\left(\max _{\Omega}|\Delta u|+\lambda_{1} \max _{\Omega}|u|\right)=: M .
\end{aligned}
$$

(Note that, without loss of generality, we assume that $M \neq 0$; otherwise, $u=0$. Therefore $F_{h}=0$ on $\Omega$.)

- $W_{4}:=\left\{\omega: u(\omega)<0,\left(\Delta+\lambda_{1}\right) u(\omega) \leq 0\right\}$. By definition,

$$
F_{h}(\omega)=\frac{\phi(\omega)}{h}\left(-u-h\left(\Delta+\lambda_{1}\right) u+u+h\left(\Delta+\lambda_{1}\right) u\right)(\omega)=0 .
$$

- $W_{5}:=\left\{\omega: u(\omega)<0,\left(\Delta+\lambda_{1}\right) u(\omega)>0,|u|<h\left(\Delta+\lambda_{1}\right) u\right\}$. Similar to the case of $W_{3},\left|F_{h}\right|<M$.

- $W_{6}:=\left\{\omega: u(\omega)<0, \Delta u(\omega)>0,|u|>\left(\Delta+\lambda_{1}\right) u h\right\}$. By definition,

$$
F_{h}(\omega)=\frac{\phi(\omega)}{h}\left(-u-h\left(\Delta+\lambda_{1}\right) u+u+h\left(\Delta+\lambda_{1}\right) u\right)(\omega)=0 .
$$

- $W_{7}:=\left\{\omega: u(\omega)=0, u_{\omega}(\omega)=0\right\}$. In this case, by definition of $\Delta|u|$, we have $\Delta|u|(\omega)=|\Delta u(\omega)|$. Therefore, $F_{h}(\omega)=0$.

2. Next, we will show that as $h \rightarrow 0, F_{h} \rightarrow 0$ almost everywhere. Fix $\omega \in \Omega \backslash I$ and consider the following cases:

- $u(\omega)>0$. We can choose $h$ small enough, such that

$$
\left|u(\omega)+h\left(\Delta+\lambda_{1}\right) u(\omega)\right|=u(\omega)+h\left(\Delta+\lambda_{1}\right) u(\omega) .
$$

Therefore,

$$
F_{h}(\omega)=\frac{1}{h} \phi(\omega)\left(u(\omega)+h\left(\Delta+\lambda_{1}\right) u(\omega)-u(\omega)-h\left(\Delta+\lambda_{1}\right) u(\omega)\right)=0 .
$$

- $u(\omega)<0$. We can choose $h$ small enough, such that

$$
\left|u(\omega)+h\left(\Delta+\lambda_{1}\right) u(\omega)\right|=-u(\omega)-h\left(\Delta+\lambda_{1}\right) u(\omega) .
$$

Therefore,

$$
F_{h}(\omega)=\frac{1}{h} \phi(\omega)\left(-u(\omega)-h\left(\Delta+\lambda_{1}\right) u(\omega)+u(\omega)+h\left(\Delta+\lambda_{1}\right) u(\omega)\right)=0 .
$$

- $u(\omega)=0$. Then as we discussed before, on $W_{7}, F_{h}(\omega)=0$.

Using 1 and 2, and the Dominated Convergence Theorem, we can conclude (34). 


\section{Proof of Lemma 4}

Let $c:=\sup _{\omega \in \Omega} \mu_{p, Q}[G(\cdot, \omega)]$. For any $\omega \in \Omega$, we have

$$
\lim _{h \rightarrow 0^{+}} \frac{1}{h} \sup _{x \neq y \in V}\left(\frac{\|x-y+h(G(x, \omega)-G(y, \omega))\|_{p, Q}}{\|x-y\|_{p, Q}}-1\right) \leq c .
$$

Fix an arbitrary $\epsilon>0$. Then there exists $h_{0}>0$ such that for all $0<h<h_{0}$,

$$
\frac{1}{h} \sup _{x \neq y \in V}\left(\frac{\|x-y+h(G(x, \omega)-G(y, \omega))\|_{p, Q}}{\|x-y\|_{p, Q}}-1\right)<c+\epsilon .
$$

Therefore, for any $x \neq y$, and $0<h<h_{0}$

$$
\frac{\|x-y+h(G(x, \omega)-G(y, \omega))\|_{p, Q}}{\|x-y\|_{p, Q}}<(c+\epsilon) h+1 .
$$

For fixed $u \neq v \in \mathbf{Y}_{V}^{(d)}$, let $\Omega_{1}=\{\omega \in \bar{\Omega}: u(\omega) \neq v(\omega)\}$. Fix $\omega_{0} \in \Omega_{1}$, and let $x=u\left(\omega_{0}\right)$ and $y=v\left(\omega_{0}\right)$. We give a proof for the case $p<\infty$; the case $p=\infty$ is analogous. Using equation (36), we have:

$$
\frac{\left(\sum_{i} q_{i}^{p} \mid u_{i}\left(\omega_{0}\right)-v_{i}\left(\omega_{0}\right)+h\left(G_{i}\left(u\left(\omega_{0}\right), \omega_{0}\right)-\left.G_{i}\left(v\left(\omega_{0}\right), \omega_{0}\right)\right|^{p}\right)^{\frac{1}{p}}\right.}{\left(\sum_{i} q_{i}^{p}\left|u_{i}\left(\omega_{0}\right)-v_{i}\left(\omega_{0}\right)\right|^{p}\right)^{\frac{1}{p}}}<(c+\epsilon) h+1 .
$$

Multiplying both sides by the denominator and raising to the power $p$, we have:

$\sum_{i} q_{i}^{p}\left|\left(u_{i}-v_{i}\right)+h\left(G_{i}\left(u, \omega_{0}\right)-G_{i}\left(v, \omega_{0}\right)\right)(\omega)\right|^{p}<((c+\epsilon) h+1)^{p} \sum_{i} q_{i}^{p}\left|\left(u_{i}-v_{i}\right)\right|^{p}$.

Since $\hat{G}(u)(\omega)=G(u(\omega), \omega)$, Equation (37) can be written as:

$$
\sum_{i} q_{i}^{p}\left|\left(u_{i}-v_{i}\right)+h\left(\hat{G}_{i}(u)-\hat{G}_{i}(v)\right)\right|^{p}<((c+\epsilon) h+1)^{p} \sum_{i} q_{i}^{p}\left|\left(u_{i}-v_{i}\right)\right|^{p} .
$$

Now by multiplying both sides of the above inequality by $\phi(\omega)$ which is nonnegative, and taking the integral over $\bar{\Omega}$, we get:

$$
\|u-v+h(\hat{G}(u)-\hat{G}(v))\|_{p, \phi, Q}<((c+\epsilon) h+1)\|u-v\|_{p, \phi, Q} .
$$

(Note that for $\omega \notin \Omega_{1}$,

$$
((c+\epsilon) h+1)^{p} \sum_{i} q_{i}^{p}\left|u_{i}(\omega)-v_{i}(\omega)\right|^{p}=0
$$

which we can add to the right hand side of (38), and also

$$
\sum_{i} q_{i}^{p}\left|u_{i}(\omega)-v_{i}(\omega)+h\left(G_{i}(u(\omega), \omega)-G_{i}(v(\omega), \omega)\right)\right|^{p}=0
$$


which we can add to the left hand side of (38), and hence we can indeed take the integral over all $\bar{\Omega}$.)

Hence,

$$
\lim _{h \rightarrow 0^{+}} \frac{1}{h}\left(\frac{\|u-v+h(\hat{G}(u)-\hat{G}(v))\|_{p, \phi, Q}}{\|u-v\|_{p, \phi, Q}}-1\right) \leq c+\epsilon .
$$

Now by letting $\epsilon \rightarrow 0$ and taking sup over $u \neq v \in \mathbf{Y}_{V}^{(d)}$, we get $\mu_{p, Q}^{+}[\hat{G}] \leq c$.

\section{Another proof of Theorem 5}

We remark here that it is also possible to prove Theorem 5 using a finite difference approximation and reducing the theorem to one about ODEs. For this end we need the following result from [2].

Proposition 1. Let $\left(x_{1}^{T}, \ldots, x_{N}^{T}\right)^{T}$ be a solution of

$$
\dot{x}_{i}=F\left(x_{i}, t\right)+D(t)\left(x_{i-1}-x_{i}+x_{i+1}-x_{i}\right), \quad i=1, \ldots, N,
$$

where $x_{0}=x_{1}, x_{N+1}=x_{N}$. For $1 \leq p \leq \infty$ and a positive diagonal matrix $Q$, let

$$
c=\sup _{(x, t)} \mu_{p, Q}\left[J_{F}(x, t)-4 \sin ^{2}(\pi / 2 N) D(t)\right] .
$$

Then

$$
\|e(t)\|_{p, Q_{p} \otimes Q} \leq e^{c t}\|e(0)\|_{p, Q_{p} \otimes Q}
$$

where $e=\left(e_{1}^{T}, \ldots, e_{N-1}^{T}\right)^{T}$ with $e_{i}=x_{i}-x_{i+1}$ and $\|\cdot\|_{p, Q_{p} \otimes Q}$ denotes the weighted $L^{p}$ norm with the weight $Q_{p} \otimes Q$, where for any $1 \leq p<\infty$,

$$
Q_{p}:=\operatorname{diag}\left(p_{1}^{\frac{2-p}{p}}, \ldots, p_{N-1}^{\frac{2-p}{p}}\right)
$$

and for $p=\infty$,

$$
Q_{\infty}=\operatorname{diag}\left(1 / p_{1}, \ldots, 1 / p_{N-1}\right)
$$

where for $1 \leq k \leq N-1, p_{k}=\sin (k \pi / N)$.

Proof of Theorem 5 Let $0=\omega_{0}<\omega_{1}<\cdots<\omega_{N+1}=L$ be the mesh points of the closed interval $[0, L]$ with equal mesh size $\Delta \omega=\frac{L}{N+1}$. For $i=0, \ldots, N+1$, define

$$
x_{i}(t):=u\left(\omega_{i}, t\right),
$$

By the Neumann boundary condition, we have:

$$
0=\frac{\partial u}{\partial \omega}(0, t) \simeq \frac{u\left(\omega_{1}, t\right)-u\left(\omega_{0}, t\right)}{\Delta \omega} \Rightarrow u\left(\omega_{1}, t\right)=u\left(\omega_{0}, t\right),
$$


Therefore for any $t, x_{0}(t)=x_{1}(t)$, and similarly, $x_{N}(t)=x_{N+1}(t)$. Now using the definition of $\partial^{2} u / \partial \omega^{2}$, we have the following expressions for $\partial^{2} u / \partial \omega^{2}$ at mesh points:

$$
\begin{aligned}
\frac{\partial^{2} u}{\partial \omega^{2}}\left(\omega_{i}, t\right) & =\lim _{\Delta \omega \rightarrow 0} \frac{u\left(\omega_{i-1}, t\right)-2 u\left(\omega_{i}, t\right)+u\left(\omega_{i+1}, t\right)}{\Delta \omega^{2}} \\
& =\lim _{N \rightarrow \infty} \frac{(N+1)^{2}}{L^{2}}\left(x_{i-1}-2 x_{i}+x_{i+1}\right)(t),
\end{aligned}
$$

we can write Equation (1) for the mesh points as follows:

$$
\begin{aligned}
\dot{x}_{1} & =F\left(x_{1}, t\right)+\frac{(N+1)^{2}}{L^{2}} D(t)\left(x_{2}-x_{1}\right) \\
\dot{x}_{2} & =F\left(x_{2}, t\right)+\frac{(N+1)^{2}}{L^{2}} D(t)\left(x_{1}-2 x_{2}+x_{3}\right) \\
\vdots & \\
\dot{x}_{N} & =F\left(x_{N}, t\right)+\frac{(N+1)^{2}}{L^{2}} D(t)\left(x_{N-1}-x_{N}\right) .
\end{aligned}
$$

Note that the ODE system (43) describes the dynamics of $N$ identical compartments that are connected through a path graph with diffusion matrix $\frac{(N+1)^{2}}{L^{2}} D(t)$. Therefore, by Proposition 1, if

$$
c_{N}:=\sup _{(x, t)} \mu_{1, Q}\left[J_{F}(x, t)-4 \sin ^{2}(\pi / 2 N) \frac{(N+1)^{2}}{L^{2}} D(t)\right],
$$

where $-4 \sin ^{2}(\pi / 2 N)$ is the smallest nonzero eigenvalue of (graph) Laplacian of a path graph, then

$$
\sum_{k=1}^{N-1} \sin (k \pi / N)\left\|\left(x_{k}-x_{k+1}\right)(t)\right\|_{1, Q} \leq e^{c_{N} t} \sum_{k=1}^{N-1} \sin (k \pi / N)\left\|\left(x_{k}-x_{k+1}\right)(t)\right\|_{1, Q} .
$$

Now dividing both sides of (44) by $\Delta \omega=\frac{L}{N+1}$ and letting $N \rightarrow \infty$, we get:

$$
\int_{0}^{L} \sin (\pi \omega)\left\|\frac{\partial u}{\partial \omega}(t)\right\|_{1, Q} d \omega \leq e^{\lim _{N \rightarrow \infty} c_{N} t} \int_{0}^{L} \sin (\pi \omega)\left\|\frac{\partial u}{\partial \omega}(t)\right\|_{1, Q} d \omega
$$

where

$$
\begin{aligned}
\lim _{N \rightarrow \infty} c_{N} & =\lim _{N \rightarrow \infty} \sup _{(x, t)} \mu_{1, Q}\left[J_{F}(x, t)-4 \sin ^{2}(\pi / 2 N) \frac{(N+1)^{2}}{L^{2}} D(t)\right] \\
& =\sup _{(x, t)} \mu_{1, Q}\left[J_{F}(x, t)-4 \lim _{N \rightarrow \infty} \sin ^{2}(\pi / 2 N) \frac{(N+1)^{2}}{L^{2}} D(t)\right] \\
& =\sup _{(x, t)} \mu_{1, Q}\left[J_{F}(x, t)-\frac{\pi^{2}}{L^{2}} D(t)\right] \\
& =c .
\end{aligned}
$$




\section{References}

[1] Z. Aminzare and E. D. Sontag. Logarithmic Lipschitz norms and diffusioninduced instability. Nonlinear Analysis: Theory, Methods and Applications, 83:31-49, 2013.

[2] Z. Aminzare and E.D. Sontag. Synchronization of diffusively-connected nonlinear systems: Results based on contractions with respect to general norms. IEEE Transactions on Network Science and Engineering, 1(2):91-106, 2014.

[3] H.G Othmer. Current problems in pattern formation. In S.A. Levin, editor, Some mathematical questions in biology, VIII, Lectures on Math. in the Life Sciences Vol. 9, pages 57-85. Amer. Math. Soc., Providence, R.I., 1977.

[4] E. Conway, D. Hoff, and J. Smoller. Large time behavior of solutions of systems of nonlinear reaction-diffusion equations. SIAM Journal on applied mathematics, 35:1-16, 1978.

[5] D. Del Vecchio, A.J. Ninfa, and E.D. Sontag. Modular cell biology: Retroactivity and insulation. Nature Molecular Systems Biology, 4:161, 2008.

[6] G. Russo, M. di Bernardo, and E.D. Sontag. Global entrainment of transcriptional systems to periodic inputs. PLoS Computational Biology, 6:e1000739, 2010 .

[7] B. Ingalls. Mathematical modeling in systems biology: An introduction. MIT Press, 2013.

[8] M. Arcak. Certifying spatially uniform behavior in reaction-diffusion pde and compartmental ode systems. Automatica, 47(6):1219-1229, 2011.

[9] A. N. Michel, D. Liu, and L. Hou. Stability of dynamical systems: continuous, discontinuous, and discrete systems. Springer-Verlag (New-York), 2007.

[10] C.A. Desoer and M. Vidyasagar. Feedback synthesis: Input-output properties. SIAM, Philadelphia, 2009.

[11] G. Dahlquist. Stability and error bounds in the numerical integration of ordinary differential equations. Inaugural dissertation, University of Stockholm, Almqvist \& Wiksells Boktryckeri AB, Uppsala, 1958.

[12] T. Strom. On logarithmic norms. SIAM J. Numer. Anal., 12:741-753, 1975.

[13] S. M. Lozinskii. Error estimate for numerical integration of ordinary differential equations. I. Izv. Vtssh. Uchebn. Zaved. Mat., 5:222-222, 1959.

[14] W. A. Coppel. Stability and asymptotic behavior of differential equations. D. C. Heath and Co., Boston, Mass., 1965.

[15] R.H Martin Jr. Bounds for solutions of a class of nonlinear differential equations. Journal of Differential Equations, 8(3):416 - 430, 1970. 
[16] G. Soderlind. The logarithmic norm. History and modern theory. BIT, 46(3):631-652, 2006.

[17] K. Deimling. Nonlinear functional analysis. Springer, 1985.

[18] Z Aminzare. On synchronous behavior in complex nonlinear dynamical systems. Dissertation, Rutgers University, The state University of New Jersey, 2015 .

[19] G. Soderlind. Bounds on nonlinear operators in finite-dimensional Banach spaces. Numer. Math, 50(1):27-44, 1986.

[20] J. Smoller. Shock waves and reaction-diffusion equations. Springer New York, New York, NY, 1994.

[21] K.P. Hadeler. Diffusion Equations in Biology. Springer Berlin Heidelberg, Berlin, Heidelberg, 2011.

[22] A. Friedman. Partial differential equations. Robert E. Krieger Publishing Co., Huntington, N.Y., original edition, 1976.

[23] D. Daners and P. Koch Medina. Abstract evolution equations, periodic problems and applications, volume 279 of Pitman Research Notes in Mathematics Series. Longman Scientific \& Technical, Harlow; copublished in the United States with John Wiley \& Sons, Inc., New York, 1992.

[24] H. Smith. Monotone dynamical systems: An introduction to the theory of competitive and cooperative systems. American Mathematical Society, 1995.

[25] R. S. Cantrell and C. Cosner. Spatial ecology via reaction-diffusion equations. Wiley Series in Mathematical and Computational Biology, 2003.

[26] J. Morgan. Boundedness and decay results for reaction-diffusion systems. SIAM J. Math. Anal., 21(5):1172-1189, 1990.

[27] A. Henrot. Extremum problems for eigenvalues of elliptic operators. Birkhauser, 2006.

[28] Z. Aminzare, Y. Shafi, M. Arcak, and E.D. Sontag. Guaranteeing spatial uniformity in reaction-diffusion systems using weighted $L_{2}$-norm contractions. In V. Kulkarni, G.-B. Stan, and K. Raman, editors, A Systems Theoretic Approach to Systems and Synthetic Biology I: Models and System Characterizations, pages 73-101. Springer-Verlag, 2014.

[29] B. Goodwin. Oscillatory behavior in enzymatic control processes. Advances in Enzyme Regulation, 3:425-439, 1965.

[30] C. Thron. The secant condition for instability in biochemical feedback control - parts i and ii. Bulletin of Mathematical Biology, 53:383-424, 1991. 